\title{
The effects of normal aging on myelinated nerve fibers in monkey central nervous system
}

\author{
Alan Peters* \\ Department of Anatomy and Neurobiology, Boston University School of Medicine, Boston, MA, USA
}

\section{Edited by:}

Kathleen S. Rockland, RIKEN Brain

Science Institute, Japan

Reviewed by:

George Bartzokis, UCLA, USA

Malin Höistad, Mount Sinai School of

Medicine, USA

\section{${ }^{*}$ Correspondence:}

Alan Peters, Department of Anatomy and Neurobiology, Boston University School of Medicine, 72 East Concord

Street, Boston, MA 02118, USA.

e-mail: valan@bu.edu
The effects of aging on myelinated nerve fibers of the central nervous system are complex. Many myelinated nerve fibers in white matter degenerate and are lost, leading to some disconnections between various parts of the central nervous system. Other myelinated nerve fibers are affected differently, because only their sheaths degenerate, leaving the axons intact. Such axons are remyelinated by a series of internodes that are much shorter than the original ones and are composed of thinner sheaths. Thus the myelin-forming cells of the central nervous system, the oligodendrocytes, remain active during aging. Indeed, not only do these neuroglial cell remyelinate axons, with age they also continue to add lamellae to the myelin sheaths of intact nerve fibers, so that sheaths become thicker. It is presumed that the degeneration of myelin sheaths is due to the degeneration of the parent oligodendrocyte, and that the production of increased numbers of internodes as a consequence of remyelination requires additional oligodendrocytes. Whether there is a turnover of oligodendrocytes during life has not been studied in primates, but it has been established that over the life span of the monkey, there is a substantial increase in the numbers of oligodendrocytes. While the loss of some myelinated nerve fibers leads to some disconnections, the degeneration of other myelin sheaths and the subsequent remyelination of axons by shorter internodes slow down the rate conduction along nerve fibers. These changes affect the integrity and timing in neuronal circuits, and there is evidence that they contribute to cognitive decline.

Keywords: rhesus monkey, oligodendrocytes, myelin sheaths, axons, degeneration, remyelination

\section{INTRODUCTION}

There are two types of nerve fibers in the central nervous system, myelinated and unmyelinated ones. The myelinated nerve fibers are axons of neurons that are ensheathed by internodal lengths of myelin formed by oligodendrocytes. Developmentally, the internodal lengths of myelin are produced at the ends of processes of oligodendrocytes and each internode is generated by a spiral wrapping of a paired sheet of oligodendrocytic plasma membrane. Initially the successive turns of the spiral of paired membrane sheets are separated by cytoplasm, but eventually the cytoplasm is extruded from between the turns. As a result, mature, compact myelin is formed. At the ends of each internodal length of myelin are regions called paranodes, and here the turns of the spiral wraps of myelin membrane successively terminate, the innermost one terminating first (Figure 1). As the turns of myelin terminate the sheath gradually becomes thinner, and eventually end at the nodes of Ranvier, which separate the successive internodal lengths of myelin. At the nodes the axon is bare, but is characterized by a dense undercoating.

An oligodendrocyte forms several internodal lengths of myelin, each one on a different axon, and in general the larger the diameter of the axon, the thicker is its myelin sheath and the longer its internodes and its paranodes (see Figure 1). And since there seems to be some limit to the amount of myelin an individual oligodendrocyte can produce and maintain, oligodendrocytes that myelinate small diameter axons form more internodal lengths of myelin than those that myelinate larger diameter axons. Myelin contains lipoproteins, so that in unfixed brains the myelin sheaths have a white sheen. Consequently, tracts of the central nervous system that contain mostly myelinated nerve fibers and few neurons are referred to as white matter. In contrast, gray matter contains the cell bodies and dendrites of neurons and fewer myelinated nerve fibers.

Our studies have been concerned with the effects of age on myelinated nerve fibers in the central nervous system of a non-human primate, the rhesus monkey (Macaca mulatta). The rhesus monkey offers an excellent model in which to examine the effects of normal aging on the brain, because unlike humans, rhesus monkeys do not develop neurofibrillary tangles are not subject to the dementia that characterizes Alzheimer's disease. In humans the existence of this disease makes it difficult to study the effects of normal aging, because it is often a problem to determine if older individuals are really normal, since they may have the beginning of Alzheimer's disease, which is characterized morphologically by the presence of both senile plaques and neurofibrillary tangles that cause neurons to die. Indeed, recent studies have shown some cognitively intact persons can have substantial numbers of plaques and tangles in their brains (e.g. Bennett, 2006; Silver et al., 2002). Added difficulties in determining which of the morphological aging changes that occur in the human brain are responsible for normal cognitive decline, is that most older people have not been behaviorally tested before their brains become available for examination, so that their real cognitive status is usually not known. And even when a brain 


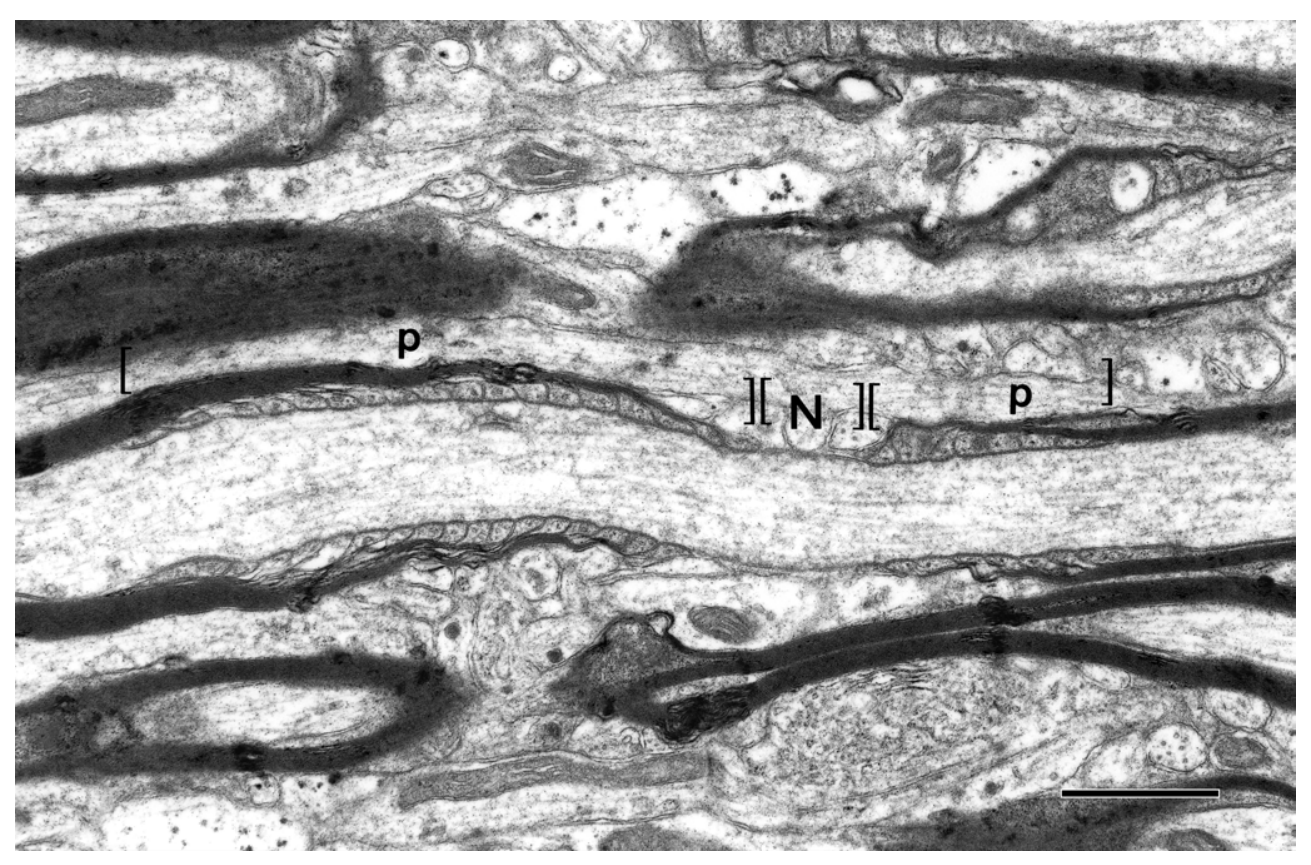

FIGURE 1 |A longitudinal section of a myelinated nerve fiber in primary visual cortex of a rhesus monkey. The section passes through a node of Ranvier (N) where the axolemma has a characteristic undercoating. On each side of the node are the paranodes (p). The sheath on the right has 8 lamellae, while the one on the left is thicker with 15 lamellae, so that it has a longer paranode. Scale bar $=1$ micron. (From Peters and Sethares, 2003). becomes available for examination, the delay in fixing the tissue usually leaves the structural preservation less than optimal.

Rhesus monkeys live for a maximum of 35 years (Tigges et al., 1988) and the advantage of using them as a model for normal aging is that they are not subject to Alzheimer's disease, and overall, do not lose significant numbers of neurons from their cerebral cortex with age (see Merrill et al., 2000; Morrison and Hof, 1997; Peters et al., 1998), However, Smith et al. (2004) have recently claimed that there may be a focal loss of neurons from cortical area $8 \mathrm{~A}$ of the prefrontal cortex. None of the neurons in the aging monkey cortex acquire neurofibrillary tangles, and although some senile plaques may be present, particularly in the frontal and primary somatosensory cortices of older monkeys, they are few in number (Heilbroner and Kemper, 1990; Struble et al., 1985). The small numbers of plaques do increase with age in monkeys, but there is no correlation between plaque burden and cognitive decline (Sloane et al., 1997). The other advantage of using monkeys to study normal cognitive decline is that over the entire range of their life span, monkeys can be behaviorally tested to determine their cognitive status, and in our studies their cognitive status is defined by a cognitive impairment index (CII). Their brains can then be properly prepared for morphological, physiological or biochemical analyses.

The first hint that there are age-related changes in myelinated nerve fibers came from the observation that in old humans and monkeys there is a decrease in the intensity of haemotoxylin staining of white matter (e.g., Kemper, 1994; Lintl and Braak, 1983). The underlying reason for this increased myelin staining pallor is still not clear, but it is now know that there are a number of age-related alterations of myelinated nerve fibers in the primate central nervous system, such as a loss of some myelinated nerve fibers and alterations in the morphology and composition of myelin sheaths, that could account for the decrease in staining intensity. Some of these alterations will be considered in the next sections, which will concentrate primarily on age changes that have been encountered in non-human primates. The correlations that occur between age-related morphological changes in myelinated nerve fibers and cognitive decline will also be considered.

\section{LOSS OF MYELINATED NERVE FIBERS}

Magnetic resonance imaging (MRI) studies of both human (e.g. Albert, 1993; Guttmann et al., 1998) and monkey (Lai et al., 1995; Wisco et al., 2008) brains have shown there is a loss of white matter from the cerebral hemispheres with age. For example, Wisco et al. (2008) calculate that in rhesus monkeys there is a $11.5 \%$ loss of white matter from the forebrain with age, in contrast to only a $2 \%$ loss of gray matter. However, it should be noted that other studies on humans (e.g. Pfefferbaum et al., 1994; Resnick et al., 2003; Sullivan et al., 2004) and monkeys (Andersen et al., 1999) suggest that loss of volume of the hemispheres is mainly due to a thinning of the cerebral cortex. Nevertheless, there seems to be general agreement that there is some loss of white matter with age and this is supported by stereological studies on cognitively normal human brains. The first of these studies was that of Pakkenberg and Gundersen (1997) who examined brains from humans between 20 and 95 years of age, and using the Cavalieri's principle to determine volume changes, they concluded there is a $28 \%$ decrease in the volume of white matter from the cerebral hemispheres. In another study from this same laboratory, Tang et al. (1997) using light and electron microscopy concluded that this loss is due to a $27 \%$ overall loss in the lengths of myelinated nerve fibers from white matter. Later Marner et al. 
(2003) extended these studies by examining samples that were taken systematically and randomly from the white matter of 36 normal brains of males and females aged between 18 and 93 years. They concluded that though the overall loss of white matter from the human cerebral hemispheres is $23 \%$, the overall decrease in total myelinated nerve fiber length is even greater, being $45 \%$. A similar loss of myelinated nerve fibers from the human brain has been reported by Meier-Ruge et al. (1992), who examined the brains of cognitively normal humans and concluded there is a $16 \%$ loss of myelinated nerve fibers from white matter of the precentral gyrus and an $11 \%$ loss from the corpus callosum.

Studies of the effects of age on the monkey brain support the contention that there is a loss of myelinated nerve fibers with age. In each of the white matter tracts we have examined some loss of myelinated nerve fibers has been found. Over the life span of the monkey the average number of myelinated nerve fibers lost from the optic nerve (Sandell and Peters, 2001), and from the anterior commissure (Sandell and Peters, 2003) is about 45\%, while from the fornix and the splenium of the corpus callosum (unpublished data), the loss is about $25 \%$. In all four structures the correlations between the decreasing numbers of myelinated nerve fibers and increasing age are significant.

In contrast, there is no measurable loss of myelinated nerve fibers from the visual cortex (Nielsen and Peters, 2000), but the inability to detect a loss may be due to the relatively sparse numbers of myelinated nerve fibers present in cortex, because a few myelinated nerve fibers with degenerating axons have been seen in cortex (Figure 2). Indeed myelinated nerve fibers with degenerating axons, as indicated in the electron microscope by the presence of dense axoplasm with a loss identifiable organelles, or the presence of empty myelin sheaths (Figure 2), have been encountered in all of the parts of the aging monkey brain that we have examined, suggesting that myelinated nerve fiber loss is ubiquitous. And based on earlier studies of Wallerian nerve fiber degeneration, there is little doubt that once an axon degenerates, breakdown and degeneration of its myelin sheath inexorably follows (e.g. Guillery, 1970).

Myelinated nerve fiber loss from white matter in pathways must result in some disconnection between various parts of the central nervous system. But interestingly, although there are no significant correlations between the extent of myelinated nerve fiber loss from the splenium of the corpus callosum and the cognitive decline shown by monkeys (Peters and Sethares, 2002), there are correlations between cognitive decline and myelinated nerve fiber loss from the anterior commissure (Sandell and Peters, 2003) and the fornix (unpublished). In this context, it is interesting that cutting the splenium of the corpus callosum, which is the principal fiber pathway connecting the occipital cortices, has little effect on cognition (Innocenti, 1986). The anterior commissure, provides the interhemispheric connection between the entire temporal lobe, as well as parts of the orbitofrontal cortex prepiriform cortex and the amygdala (Demeter et al., 1990; Jouandet and Gazzaniga, 1979; Sullivan and Hamilton, 1973a,b), and numerous studies have shown that the anterior commissure provides a pathway whereby visual information can reach the opposite hemisphere and contribute to behavioral responses, such as two-choice discrimination (Doty et al., 1994; Gross et al., 1977; Sobotka and Ringo, 1996). The fornix, on the other hand, carries the main output from the

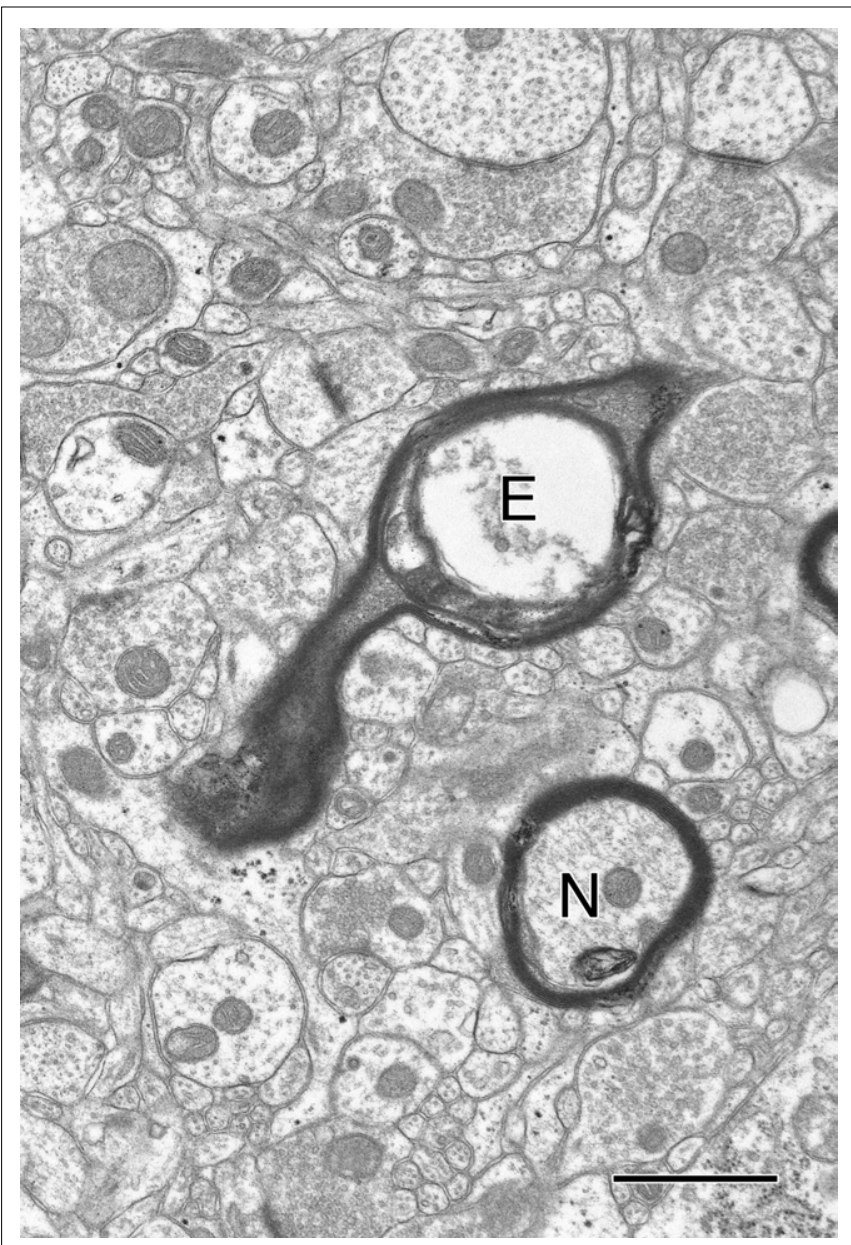

FIGURE 2 | In the field is a normal nerve fiber (N) and another nerve fiber $(E)$ in which the axon has degenerated, leaving an empty sheath. Layer 4 from area 46 of a 27 -year-old rhesus monkey. Scale bar $=1$ micron.

hippocampus, and studies of the effects of lesioning the fornix in both monkeys (Fletcher et al., 2006; Gaffan et al., 2001; Owen and Butler, 1981; Wilson et al., 2007) and humans (e.g. D'Esposito et al., 1995; Gaffan et al., 1991) have revealed the role of the fornix in memory and have described amnesia as a major consequence of making such lesions.

It might be assumed that since myelinated nerve fibers are lost from white matter with age, that there must be a concomitant loss of the neurons from which the nerve fibers arise. For the optic nerve, this may be the case, since retinal ganglion cells are subject to damage from ocular changes and systemic disease that occurs frequently in the elderly (Garner et al., 1994). But for the other central nervous system pathways, in which the myelinated nerve fibers arise from cortical neurons, a different reason has to be sought, because, as stated above, recent studies have shown that in normal aging few neurons are lost from the cerebral cortices of either monkeys or humans (e.g., Hof et al., 2000; Merrill et al., 2000; Morrison and Hof, 1997; Pakkenberg and Gundersen, 1997; Peters et al., 1998), and Freeman et al. (2008) have recently shown that in normally aging humans cortical neuron numbers are preserved even when there is cortical atrophy. To account for the age-related loss of myelinated 
nerve fibers from white matter, we have suggested that only the portion of the axonal plexus of a pyramidal cell that enters the white matter, degenerates by a dying back process, leaving the more extensive local axonal plexus in the cortex intact (Peters and Rosene, 2003). This scenario would account both for the loss of some myelinated nerve fibers from white matter and for the failure to detect myelinated nerve fiber loss from the cerebral cortex itself.

\section{DEGENERATIVE CHANGES IN MYELIN SHEATHS}

Obviously, in normal aging some myelin sheaths degenerate as a consequence of their axons degenerating, but in other cases myelin sheaths degenerate even though the axon is intact. In the latter category there are two kinds of myelin sheath alterations. The most common age-related degenerative alteration is an accumulation of dark cytoplasm in pockets that are produced by a splitting of the major dense line (e.g. Peters et al., 1994; Peters and Sethares, 2002; Peters et al., 2000; Sandell and Peters, 2003). Examples of what will be referred to as dense sheaths are shown in Figure 3. The location of the dense cytoplasm in splits of the major dense line implies that the cytoplasm must be derived from the parent oligodendrocyte, because the major dense line of the myelin sheath is produced by apposition of the cytoplasmic faces of the plasma membrane of the oligodendrocyte forming the myelin sheath. The amount of dense cytoplasm can vary from a small amount contained in a local split of the sheath to an accumulation that is extensive, causing the sheath to bulge out into the surrounding intercellular space. Longitudinal sections of affected sheaths show that the accumulations of dense cytoplasm are localized, although there may be several such loci along an internodal length of myelin. Proof of the fact that the accumulation of dense cytoplasm in normal aging is a degenerative change comes from studies of Curprizone toxicity, which leads to oligodendrocyte death, resulting in the formation of dense cytoplasm in the cytoplasmic process on the inner face of the myelin sheath (Ludwin, 1978). A similar dense cytoplasm also occurs in the sheaths of mice with a myelin-associated glycoprotein deficiency (e.g. Lassmann et al., 1997).

Another, but less common myelin alteration associated with aging is the formation of myelin balloons (e.g. Feldman and Peters, 1998; Peters and Sethares, 2003). These balloons can be as large as $10 \mu \mathrm{m}$ in diameter, so that even by light microscopy the larger balloons they are visible as holes in the neuropil of the aging cortex. Electron microscopic analyses show that these holes are really localized fluid-filled cavities that are accommodated by splits in the intraperiod line of the affected sheaths, and since the intraperiod line is produced by apposition of the outer faces of the cytoplasmic membrane of the oligodendrocyte, the fluid-filled sacs are potentially in contact with the extracellular space. In larger balloons, the axon of the nerve fiber is pushed to one side of the sheath,

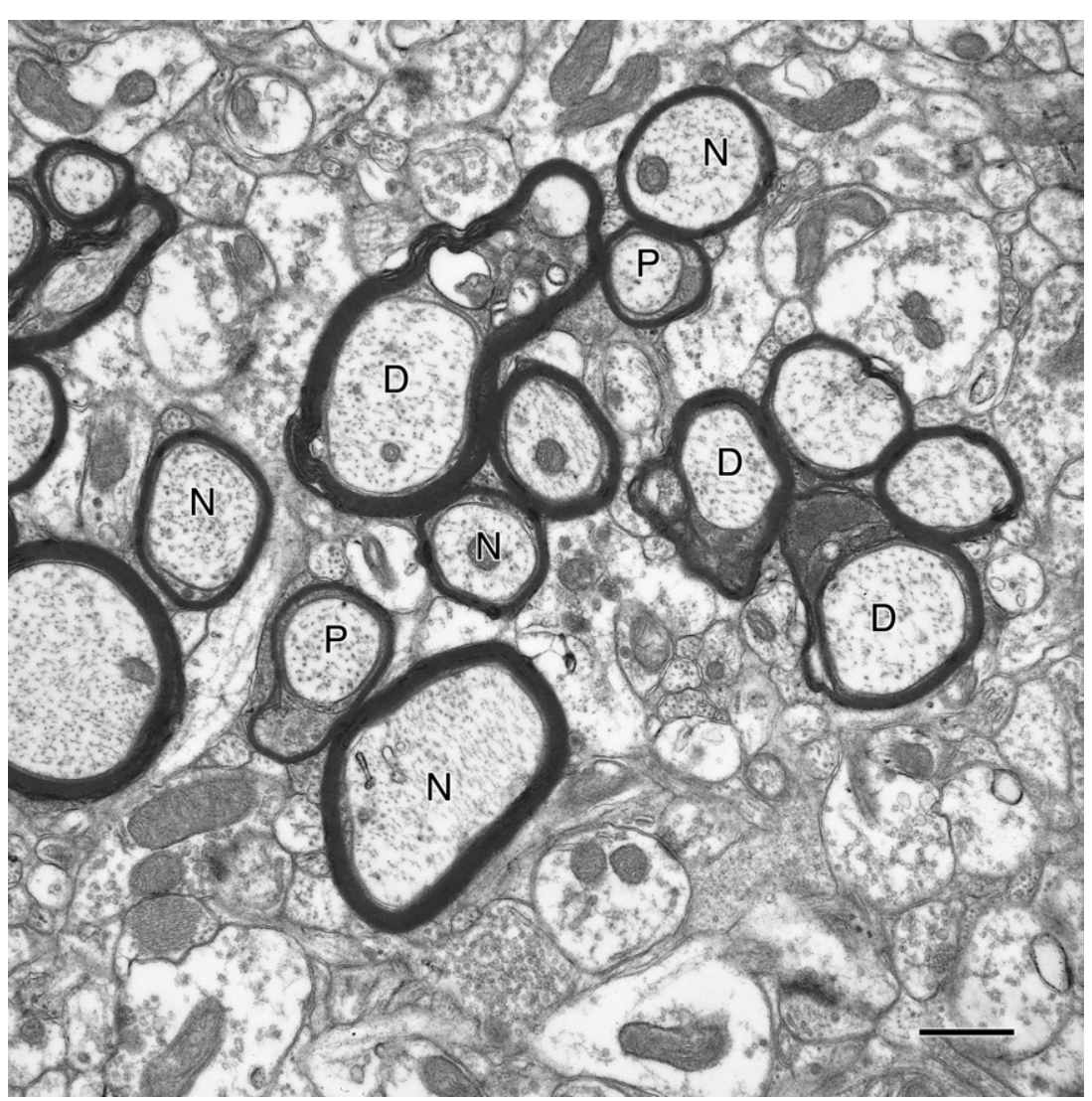

FIGURE 3 |A cross-sectioned nerve fiber bundle in primary visual cortex of a 29-year-old rhesus monkey. In this bundle some of the nerve fibers (N) have normal sheaths and are sectioned through internodes, and others $(P)$ are sectioned through paranodes. Three nerve fibers (D) have degenerating sheaths, as shown by the accumulation of dense cytoplasm in splits between lamellae. Scale bar $=1$ micron. 
suggesting that the fluid in the sac must be exerting some pressure. But despite this, the sheath is of the same thickness all around the balloon, and there is no obvious change in the periodicity of the myelin lamellae. Consequently it does not appear that the myelin sheath is elastic, and so the formation of a balloon must entail the production of excess myelin by the parent oligodendrocyte. It should be emphasized that dense sheaths and balloons are not entirely separate entities, since it is not uncommon for a balloon to have some dense cytoplasm at its base, or for a sheath with a balloon to have some dense cytoplasm between its lamellae.

Again, there is evidence that the formation of balloons is a degenerative process, since myelin balloons can be produced by Cuprizone (Ludwin, 1978) and tetraethyl tin (Malamud and Hirano, 1973) toxicity, and by chronic copper poisoning (Hull and Blakemore, 1974). Balloons can also occur in early phases of Wallerian degeneration (Franson and Ronnevi, 1989), and in severe diabetes (Tamura and Parry, 1994).

When the percentage of myelinated nerve fibers showing either the presence of dense cytoplasm or of balloons is examined, it is found that the frequency of such profiles increases significantly with age (e.g. Feldman and Peters, 1998). More importantly there are significant correlations between cognitive declines and the frequency of profiles of degenerating sheaths in cortical area 46 (Peters and Sethares, 2002), splenium of the corpus callosum (Peters and Sethares, 2002), anterior commissure (Sandell and Peters, 2003), and fornix (unpublished data). An exception is primary visual cortex, in which there is no correlation between cognitive decline and myelin sheath degeneration (Peters et al., 2000). This may be because primary visual cortex has little role in cognition. It is presumed that the correlations between myelin degeneration and cognition are due to the degenerating slowing down conduction velocity, and thus affecting the timing in neuronal circuits.

Duce et al. (2007) have identified a number of genes that might produce cytotoxicity in white matter. These genes range from ones that can affect life span, to ones that can affect the reorganization of glial cytoskeleton, others that can produce oxidative and proteolytic injury, and yet others that are cell cycle inhibitors. But these authors focus particular attention on a gene called Klotho, a multifunctional gene that is known to defend against oxidative stress, and suggest that with a decrease in the activity of Klotho there is a loss of this protection, which may result in the death of oligodendrocytes.

\section{THE CONTINUED FORMATION OF MYELIN}

There are other age-related alterations in myelin sheaths, which indicate that myelin continues to form with age. The first is an increase in the overall thickness of normal myelin sheaths with age. Thus, in the primary visual cortex of the monkey the mean number of lamellae in sheaths of myelinated nerve fibers in layer $4 \mathrm{C} \beta$ of young monkeys is 5.6 , while in old monkeys it is 7.0 (Peters et al., 2001). However, the increase in thickness of sheaths is not uniform, because the mean increase in the numbers of lamellae is largely because thick sheaths, with more than 10 lamellae, become more common in old monkeys. This increase in the numbers of lamellae on nerve fibers with thicker myelin sheaths often affects their paranodes, so that whereas longitudinal sections of young nerve fibers show the paranodal pockets of cytoplasm to terminate is a regular sequence and to be all in contact with the underlying axolemma (see Figure 1), in nerve fibers of old monkeys with thicker sheaths the paranodes can be disarrayed. They can be piled up on one another, so that only some of the paranodal loops are in contact with the axolemma (Hinman et al., 2006). A similar situation has been reported in the myelinated nerve fibers of old rats (Sugiyama et al., 2002), and it seems likely that such disruption of the paranodal region could affect conduction velocity. As yet, not determination seems to have been made about whether myelin sheaths in white matter also become thicker with age.

Another change that is considered to indicate the continued formation of myelin is the formation of sheaths that contain redundant myelin, so that the sheaths are too large for their enclosed axons. When such sheaths are cross-sectioned and examined by electron microscopy the axon is seen to be located at one end of an excessively large sheath that loops off into the surrounding neuropil. Such sheaths were first described by Rosenbluth (1966) in the cerebellum of the toad. Sturrock (1976) described such sheaths in anterior commissures of old mice, and later Cullen and Webster (1979) found them to be common in the optic nerves of metamorphosing toads. During metamorphosis the optic nerves become shorter, and the myelin sheaths undergo extensive remodeling, producing redundant sheaths that disappear later in development. These events led Cullen and Webster to suggest that the overproduction of myelin is to allow the sheaths to enlarge so that they can accommodate subsequent increases in the diameters of the enclosed axons. However, the role of redundant sheaths in the aging process is not yet evident, although axons in these sheaths generally have small diameters.

\section{REMYELINATION}

When the frequencies of various kinds of profiles of myelinated nerve fibers are quantified in the vertical bundles of nerve fibers in the cerebral cortex it becomes evident that the frequency of profiles of paranodes increases with age (Peters and Sethares, 2003). As pointed out earlier, for the present purposes the paranodes are defined as those regions at the ends of internodes where the spiraled myelin lamellae gradually terminate as the sheath approaches a node of Ranvier. As the myelin lamellae terminate, the major dense line opens up to accommodate a spiral of cytoplasm, which in longitudinal sections through paranodes appears as a series of pockets of cytoplasm on each side of the axon (see Figure 1). Where the plasma membrane on the inner faces of these pockets meet the axolemma, the two membranes, become very close to each other and form a junctional complex. This membrane apposition makes it quite easy to identify profiles of myelinated nerve fibers sectioned through paranodes (Figure 3), and it turns out there is a $57 \%$ increase in the frequency of paranodal profiles in the aging visual cortex, a $90 \%$ increase in area 46 of prefrontal cortex (Peters and Sethares, 2003), and a 60\% increase in the anterior commissure (Sandell and Peters, 2003).

There could be two reasons for these age-related increases in paranodal profiles. One is an increase in the lengths of the paranodes with age. The second is an overall increase in the numbers of paranodes. Paranodes do become slightly longer as the numbers of lamellae in myelin sheaths increase with age, but not enough to account for the $60 \%$ or more increase in the frequency of paranodal profiles. Consequently, the increase in frequency has to be due to an increase in the overall numbers of internodal lengths of myelin. This 
would occur if remyelination were taking place, such that some of the original internodal lengths of myelin degenerate and are replaced by shorter internodes. The accepted hallmarks of remyelination are the presence of short internodes and of sheaths that are inappropriately thin for the size of the enclosed axons (e.g. Brück et al., 2003; Hirano, 1989; Kreutzeberg et al., 1997; Ludwin, 1995; Prineas and McDonald, 1997). Such internodes have been found in the aging central nervous system. Internodes of myelin, as short as 3-8 $\mu \mathrm{m}$, are present in old monkeys, as well as sheaths that are inappropriately thin for the size of the enclosed axon (see Peters and Sethares, 2003).

We have not been able to identify demyelinated nerve fibers in the monkey brain, but this should not be a surprise, since such demyelinated nerve fibers would be expected to resemble unmyelinated nerve fibers. However, in support of the fact that demyelination is taking place, we have seen fragments of degenerating myelin within the cytoplasm of both microglia, and more commonly within astrocytes in the brains of aging monkeys. Also some of the amorphous phagocytosed material within the cytoplasm of astrocytes in the cerebral cortex of old monkeys labels for antibodies to myelin basic protein (Peters and Sethares, 2003).

A recent article on the remyelination of rubrospinal nerve fibers that remyelinate after a contusion lesion of the spinal cords of mice serves to shed some light on what is happening during aging. Lasiene et al. (2008) have shown that remyelinated nerve fibers in the rubrospinal tract of mice have much shorter internodal lengths than in control mice, and that these remyelinated axons conduct at a lower rate than the controls. There is also evidence that there are reductions in conduction velocity in the nerve fibers of aging cats (Morales et al., 1987; Xi et al., 1999). A reduction in conduction velocity also occurs in demyelinating diseases (e.g. Felts et al., 1997; Waxman et al., 1995).

Consequently, it can be assumed that remyelinated nerve fibers with shorter internodes in the aging monkey also have a slower conduction rate than the nerve fibers that remain unaffected by age, and that this would affect the timing in neuronal circuits (e.g. see Wang et al., 2005). However, when correlations between the frequency of occurrence of profiles of paranodes and the overall cognitive status of individual monkeys, as measured by the Cognitive Impairment Index, CII, are examined, there is no significant correlation between the two measures, for visual cortex and anterior commissure, but there is a correlation for area 46 of prefrontal cortex $(p<0.01$; Peters and Sethares, 2002). The reason for this correlation between CII and paranodal frequency may have to do with the unique role of prefrontal cortex in cognition.

\section{EFFECTS OF AGE ON OLIGODENDROCYTES}

In monkey cerebral cortex stained with Perl's reaction for ferric iron the processes of some oligodendrocytes in old monkeys show swellings along their lengths (Figure 4; insert), and when these swellings

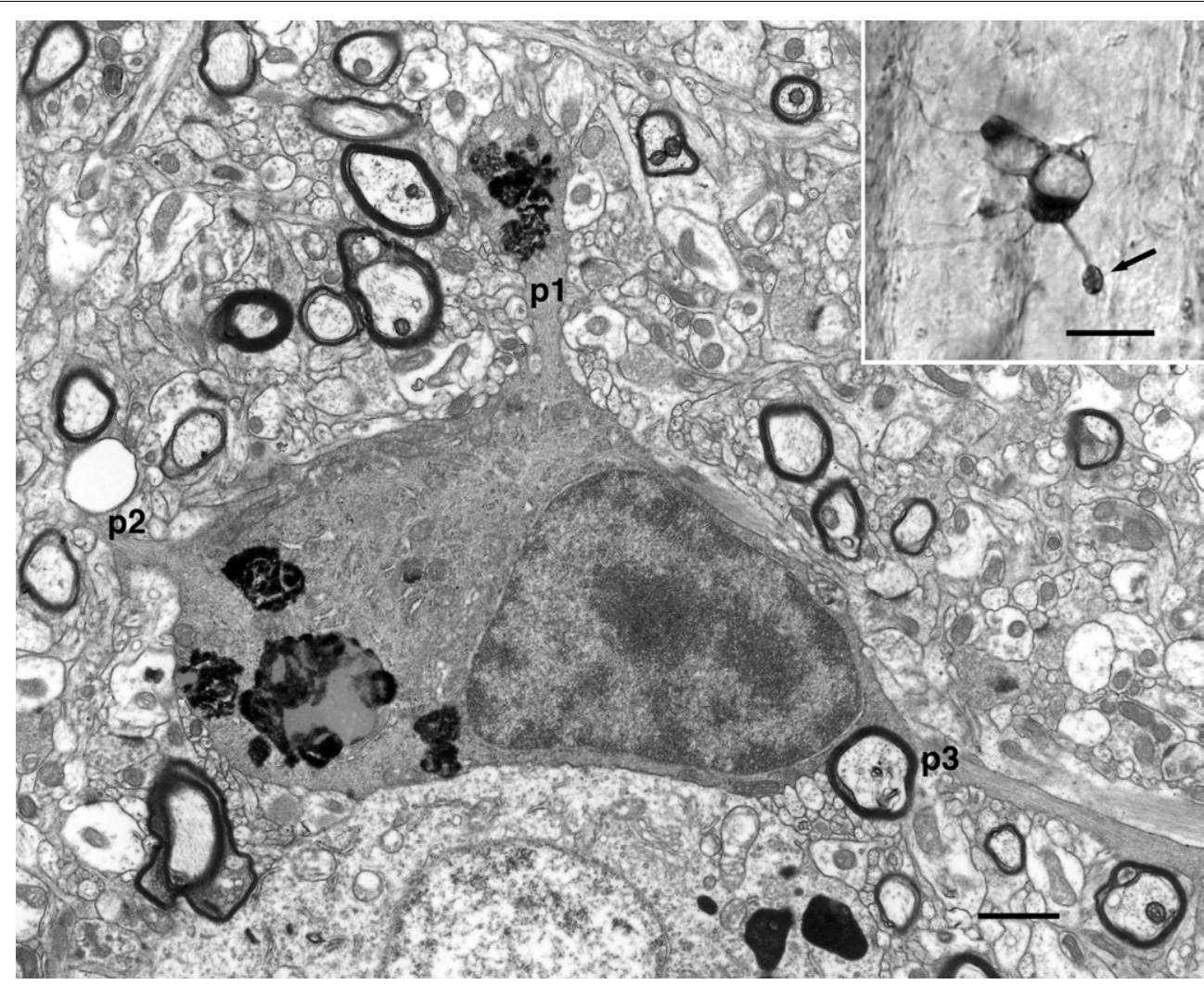

FIGURE 4 | Electron micrograph of an oligodendrocyte in layer 6 of area 17 of a 35-year-old rhesus monkey. Three processes, p1-p3, extend from the cell body. One of the processes, p1, has a swelling that contains dense inclusions, which are similar to the dense inclusions in the cell body. Scale bar $=1$ micron.
The insert shows a light microscopic image of an oligodendrocyte stained with Perl's reaction for iron compounds. Note the large swelling (arrow) on one of the processes. It is similar to the one seen on process $\mathrm{p} 1$ in the accompanying electron micrograph. Area 46 of a 28-year-old monkey. Scale bar $=10$ microns 
are examined in the electron microscope it is seen that they contain dense inclusions (Figure 4). Similar dense inclusions also occur in the perikarya of old oligodendrocytes (Peters, 1996; Peters and Sethares, 2004; Peters et al., 1991), and since the dense material is not membrane bound, it is unlikely to be produced by phagocytosis. Similar swellings along oligodendrocyte processes have been reported in twitcher mice, which are a model for globoid leukodystrophy, and Levine and Torres (1992) suggest that the material in the swellings comes from components of myelin sheaths that are being renewed. Most probably the material is produced by degeneration of some components of the myelin sheaths that belong to the oligodendrocytes, and it is tempting to suggest that the material is related to the dense cytoplasm that accumulates between the lamellae of some sheaths in old monkeys.

It is also common in old monkeys to find oligodendrocytes in pairs, rows and groups, suggesting that oligodendrocytes may be proliferating with age (Peters and Sethares, 2004; Peters et al., 1991), and when comparisons are made between the numbers of oligodendrocytes in young and old primary visual cortices it is evident that there is an increase in the numbers of oligodendrocytes with age (Peters et al., 1991). Thus in layer 4C $\beta$ for example, in which oligodendrocytes account for about $55 \%$ of the total population of neuroglial cells, there is a $50 \%$ increase in the numbers of oligodendrocytes with age (Peters and Sethares, 2004). In a more recent study an assessment was made of the effects of age on the populations of neuroglial cells throughout the depth of monkey primary visual cortex (Peters et al., 2008). It was seen that the numbers of oligodendroglial cells in the various layers essentially reflects the frequency of myelinated nerve fibers within them, the greatest numbers of oligodendrocytes being in the deeper layers. Again, with age the numbers of oligodendrocytes in all layers was found to increase by about $50 \%$. In contrast, there are no changes in the frequency of either astrocytes or microglial cells with age. There is also an increase in the frequency of oligodendrocytes in monkey optic nerve with age (Sandell and Peters, 2002), as well as in the fornix (unpublished data), but not in the anterior commissure (Sandell and Peters, 2003). The reason for this difference is not yet apparent.

What is the origin of the increased numbers of oligodendrocytes that are generated, and why are they necessary? The formation of groups and rows of oligodendrocytes during aging could be taken to suggest that oligodendrocytes are dividing, but the prevailing view is that mature oligodendrocytes do not divide (see Keirstead and Blakemore, 1997; Ludwin, 1995; Norton, 1994), and in a study of the generation of new cells in the adult dentate gyrus of the hippocampus in old monkeys using BrdU labeling no labeled oligodendrocytes were found (Ngwenya et al., 2008). It is more likely that new oligodendrocytes originate from the oligodendroglial precursor cells which express NG2 chondroitin sulfate, These cells are scattered throughout the central nervous system, and in adult rodents they account for about $5 \%$ of all neuroglial cells (e.g. Levine et al., 2001).

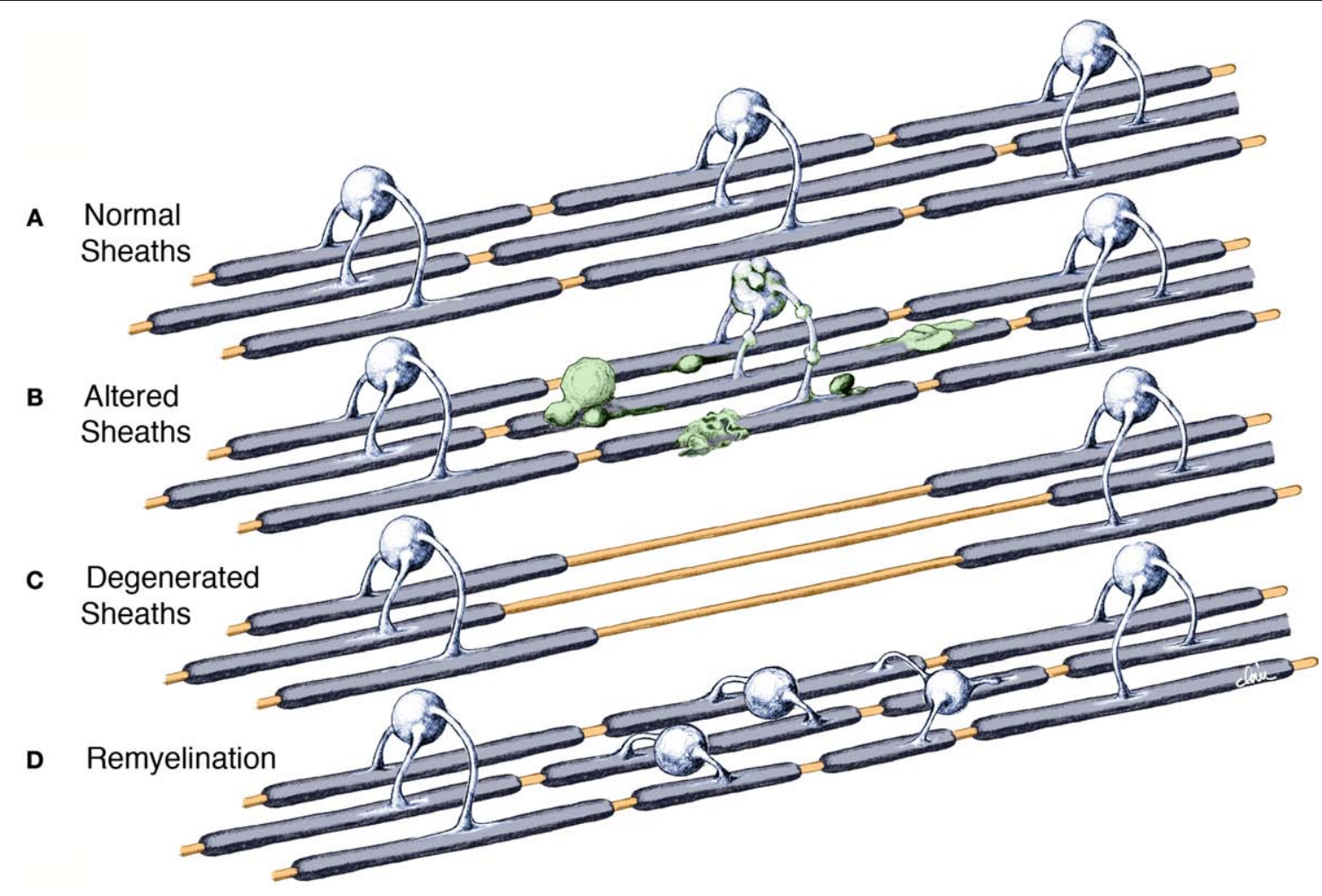

FIGURE 5 | Diagrammatic representation of the degeneration of sheaths with age, and the subsequent remyelination of axons. (A) Normal state. (B) Some sheaths become altered by the presence of dense cytoplasm and the formation of balloons. This is believed to occur when the oligodendrocyte accumulates dense inclusions within its cell body and within swellings along its processes. (C) The degeneration of myelin sheaths leaves axons bare. (D) The bare axons are remyelinated by newly generated oligodendrocytes that form short internodal lengths with thin sheaths. 
Studies such as that by Cerghet et al. (2006) have shown that there is a turnover of oligodendroglial cells in the adult mouse, such that some oligodendrocytes undergo apoptosis, and die, while new oligodendrocytes are being generated. And interestingly, Cerghet et al. (2006) found the turnover of oligodendrocytes in the corpus callosum to be greater in female mice than in males, indicating that the lifespan of oligodendrocytes is shorter in females than in males. Moreover, Rivers et al. (2008) have recently shown that in adult mice many of the newly generated oligodendrocytes that arise from the oligodendrocytic precursor cells during adult hood are involved in myelination. They calculate that about $20 \%$ of all oligodendrocytes in the adult corpus callosum are generated during adulthood and that many of these cells form myelin. In contrast, the same group calculates that only about $5 \%$ of the adult-born oligodendrocytes in the cerebral cortex appear to be involved in the elaboration of myelin sheaths, but Rivers et al. (2008) were not able to determine the function of the other adult generated oligodendrocytes present in cortex.

Unfortunately there is no information about the rate of turnover of oligodendrocytes in the adult monkey, but there is no reason to doubt that it is basically different from in rodents.

\section{A SYNTHESIS}

It is proposed that the following scenario can explain the available data on the effects of age on myelinated nerve fibers in the central nervous system of the monkey. During aging some neurons lose their long projecting myelinated axons that enter white matter, while retaining their local plexuses so that the parent neuron does not die. The consequence of this is that, as has been demonstrated, some myelinated nerve fibers are lost from white matter, even though there is no significant loss of neurons from the cerebral cortex. For other neurons the effects of aging are less severe (see Figure 5), since their axons remains intact, even though some of

\section{REFERENCES}

Albert, M. (1993). Neuropsychological and neurophysiological changes in healthy adult humans across the age range. Neurobiol. Aging 14, 623-625.

Andersen, A. H., Zhang, Z., Gash, D. M., and Avison, M.J.(1999).Age-associated changes in CNS composition identified by MRI. Brain Res. 829, 90-98.

Bennett, D. A. (2006). Postmortem indices linking risk factors to cognition: results from the Religious Order Study and the Memory and Aging Project. Alzheimer Dis. Assoc. Disord. 20, S63-S68.

Brück, W., Kuhlmann, T., and Stadelmann, C. (2003). Remyelination in multiple sclerosis. J. Neurol. Sci.206, 181-185.

Cerghet, M., Skoff, R. P., Bessert, D., Zhang, Z., Mullins, C., and Ghandour. M.S. (2006). Proliferation and death of oligodendrocytes and myelin proteins are differentially regulated in male and female rodents. J. Neurosci. 26, 1439-1447.
Cullen, M. J., and Webster, H. D. (1979). Remodelling of optic nerve sheaths and axons during metamorphosis in Xenopus laevis. J. Comp. Neurol. 184, 353-362.

Demeter, S., Rosene, D. L., and Van Hoesen, G. W. (1990). Fields of origin and pathways of the interhemispheric commissures in the temporal lobe of macaques. J. Comp. Neurol. 302, 29-53.

D’Esposito, M., Verfaelie, M., Alexander, M. P., and Katz, D. I. (1995). Amnesia following traumatic bilateral fornix transection. Neurology 45, 1546-1550.

Doty, R. W., Ringo, J. L., and Lewine, J. D. (1994). Interhemispheric sharing of visual memory in macaques. Behav. Brain Res. 64Z, 79-84.

Duce, J. A., Podvi, S., Hollander, W., Kipling, D., Rosene, D. L., and Abraham, C. R. (2007). Gene profile analysis implicates Klotho as an important contributor to aging changes in brain white matter of the rhesus monkey. Glia 56, 106-117.

the internodal lengths of myelin that ensheath them degenerate (Figure 5B). The process of demyelination probably begins as an oligodendrocyte shows stress and starts to accumulate dense inclusions in swellings of its processes and in its perikaryon, as well as in spaces between the lamellae of the myelin sheaths for which the oligodendrocyte is responsible. Ultimately the oligodendrocyte dies, which results in the degeneration and loss of the internodal lengths of myelin belonging to that oligodendrocyte (Figure 5C). Oligodendrocyte precursor cells are then activated and generate new oligodendrocytes that repair the damage by remyelinating the bare lengths of axons. In the process of remyelination, several new oligodendrocytes are involved in the replacement of the original internode of myelin. These oligodendrocytes produce shorter internodal lengths than the original one, and the new sheaths are thinner (Figure 5D). Thus, when profiles of sectioned myelin sheaths in older monkeys are examined, it is found there is an increase in the number of profiles of paranodes, and this is accompanied by an increase in the total number of oligodendrocytes. This breakdown of myelin sheaths, together with the formation of shorter internodal lengths of myelin and the consequent increase in the number of nodes of Ranvier, would result in a slowing down of the rate of conduction along affected myelinated nerve fibers. Consequently the timing in neuronal circuits would be affected and contribute to cognitive impairment that occurs with increasing age.

\section{ACKNOWLEDGEMENTS}

The author wishes to thank Claire Folger for her continued help and expert technical assistance during the performance of these studies on the aging brain. I am also grateful for the figure that she produced of the changes that affect myelin sheaths during aging. This work was funded by the National Institutes of Health, National Institute on Aging, Program Project Grant 1P0 AG000001.

Feldman, M. L., and Peters, A. (1998) Ballooning of myelin sheaths in normally aged macaques. J. Neurocytol. 27, 605-614.

Felts, P. A., Baker, R. A., and Smith, K. J. (1997). Conduction along segmentally demyelinated mammalian central axons. J. Neurosci. 17, 7267-7277.

Fletcher, B. R., Calhoun, M.E., Rapp, P. R., and Shapiro, M. L. (2006). Fornix lesions decouple the induction of hippocampal arc transcription from behavior but not plasticity. J. Neurosci. 26, 1507-1515.

Franson, P., and Ronnevi, L.-O. (1989). Myelin breakdown in the posterior funiculus of the kitten after dorsal rhizotomy: a qualitative light and electron microscopic study. Anat. Embryol. 180, 273-280.

Freeman, S. H., Kandel, R., Cruz, L., Rozkaine, A., Newell, K., Frosch, M. P., Hedley-White, T., Locascio, J. J., and Hyman, B. T. (2008). Preservation of neuronal number despite agerelated cortical brain atrophy in elderly subjects without Alzheimer disease. J. Neuropathol. Exp. Neurol. 67, 1205-1212.

Gaffan, E. A., Gaffan, D., and Hodges, J. R. (1991). Amnesia following damage to the left fornix and other sites. A comparative study. Brain 114, 1297-1313.

Gaffan, E. A., Parker, A., and Easton, A (2001). Dense amnesia in the monkey after transection of fornix, amygdala and anterior temporal stem. Neuropsychologica 39, 51-70.

Garner, A., Sarks, S., and Sraks, J. P. (1994). Degenerative and related disorders of the retina and choroid. In Pathobiology of Ocular Disease; A Dynamic Approach, A. Garner and G. K. Klintworth, eds (New York, Marcel Decker), pp. 631-674.

Gross, C. G., Bender, D. B., and Mishkin, M., (1977). Contributions of the corpus callosum, and the anterior commissure to visual activation of inferior temporal neurons. Brain Res. 131, 227-239.

Guillery, R. W. (1970). Light- and electron-microscopical studies of 
normal and degenerating axons. In Contemporary Research Methods in Neuroanatomy, W. J. H. Nauta and S. O. E. Ebbesson, eds (New York, Springer), pp. 77-105.

Guttmann, C.R.G., Jolesz, F.A., Kikinis, R., Killiany, R. J., Moss, M. B., Sandor, T., and Albert, M.S. (1998). White matter changes with normal aging. Neurology 50, 972-978.

Heilbroner, P.L., and Kemper, T.L. (1990), The cytoarchitectonic distribution of senile plaques in three aged monkeys. Acta Neuropathol. 81, 60-65.

Hinman, J. D., Peters, A., Cabral, H., Rosene, D. L., Hollander, W., Rasband, M. N., and Abraham, C. R. (2006). Age-related molecular reorganization at the node of Ranvier. J. Comp. Neurol. 495, 351-362.

Hirano, A. (1989). Review of the morphological aspects of remyelination. Dev. Neurosci. 11, 112-117.

Hof, R.P., Nimchinsky, E.A., Toung, W. G., and Morrison, J.H. (2000). Numbers of Meynert and layer IVB neurons in area V1: a stereologic analysis in young and aged macaque monkeys. J. Comp. Neurol. 420, 113-126.

Hull, J. M., and Blakemore, W. F. (1974). Chronic copper poisoning and changes in the central nervous system of sheep. Acta Neuropathol. 29, 9.

Innocenti,G.M. (1986). General organization of callosal connections in the cerebral cortex. In Sensory-Motor Areas and Aspects of Cortical Conectivity. Cerebral Cortex, Vol. 5, E. G. Jones and A. Peters, eds (New York, Plenum Press), pp. 291-354.

Jouandet, M. L., and Gazzaniga, M. S. (1979). Cortical fields of origin of the anterior commissure of the rhesusmonkey. Exp. Neurol. 66, 381-397.

Keirstead, H. S., and Blakemore, W. F. (1997). Identification of post-mitotic oligodendrocytes incapable of remyelination within demyelinated adult spinal cord. J. Neuropathol. Exp. Neurol. 56, 1191-1201.

Kemper, T. L. (1994). Neuroanatomical and neuropathological changes during aging and dementia. In Clinical Neurology of Aging, M. Albert and J. E. Knoefel, eds (New York: Oxford University Press), pp. 3-67.

Kreutzeberg, G., Blakemore, W. F., and Graeber, M. B. (1997). Cellular pathology of the central nervous system. In Greenfield's Neurophology. 6th Edn, D. I. Graham and P. L. Lantos, eds (London, Arnold), pp. 85-156.

Lai, Z. C., Rosene, D. L., Killiany, R. J., Pugliese, D., Albert, M. S., and Moss, M. B. (1995). Abstr. - Soc. Neurosci. 21, 1564.

Lasiene, J., Shue, L., Perlmutter, S., and Horner, P. (2008). No evidence for chronic demyelination in spared axons after spinal cord injury in a mouse. J. Neurosci. 28, 3887-3896.

Lassmann, H., Bartsch, U., Montag, D., and Schachner, M. (1997). Dying-back oligodendrogliopathy; a late sequel of myelin-associated glycoprotein deficiency. Glia 19, 104-110.

Levine, J. M., Reynolds, R., and Fawcett, J W. (2001). The oligodendrocyte precursor cell in health and disease. Trends Neurosci. 24, 39-47.

Levine, S. M., and Torres, M. V. (1992). Morphological features of degenerating oligodendrocytes in twitcher mice. Brain Res. 587, 348-352.

Lintl, P., and Braak, H. (1983). Loss of intracortical myelinated fibers: a distinctive age-related alteration in the human striate area. Acta Neuropathol. 61, 178-182.

Ludwin, S. K. (1978). Central nervous system demyelination and remyelination in the mouse. An ultrastrructural study of cuprizone toxicity. Lab. Invest. 39, 597-612.

Ludwin, S. K. (1995). Pathology of the myelin sheath. In The Axon: Structure, Function and Pathophysiology, S. G. Waxman, J. D. Kocsis, and P. K. Stys, eds (New York, Oxford University Press), pp. 412-437.

Malamud, N., and Hirano, A. (1973). Atlas of Neuropathology. Berkeley, University of California Press.

Marner, L., Nyengaard, J. R., Tang, Y., and Pakkenberg. B. (2003). Marked loss of myelinated nerve fibers in the human brain with age. J. Comp. Neurol. 462, 144-152.

Meier-Ruge,A., Ulrich, J., Bruhlmann, M., and Meier, E. (1992). Age-related white matter atrophy in the human brain. Ann. N.Y. Acad. Sci. 673, 260-269.

Merrill,D.A., Roberts, J.A.S., and Tuszyski, M.H. (2000). Conservation of neuron numbers and size in entorhinal cortex layers II, III, and V/VI of aged primates. J. Comp. Neurol. 422, 396-401.

Morales, F. R., Boxer, P. A., Fung, S. J., and Chase, M. H. (1987). Basic electrophysiological properties of spinal cord motoneurons during old age in the cat. J. Neurophysiol. 58, 180-195.

Morrison, J. H., and Hof, P. R. (1997). Life and death of neurons in the aging brain. Nature 278, 412-419.

Ngwenya, L. B., Rosene, D. L., and Peters, A. (2008). An ultrastructural study of the newly generated cells in the adult monkey dentate gyrus. Hippocampus 18, 210-220.

Nielsen, K., and Peters, A. (2000). The effects of aging on the frequency of nerve fibers in rhesus monkey striate cortex. Neurobiol. Aging 21, 621-628.

Norton, W. T. (1996). Do oligodendrocytes divide? Neurochem. Res. 21, 495-503.

Owen, N. J., and Butler, S. R. (1981). Amnesia after transection of the fornix in monkeys; long-term memory impaired, short-term memory intact. Behav. Brain Res. 3, 495-503.

Pakkenberg, B., and Gundersen, H. J. G. (1997). Neocortical neuron number in humans: effects of sex and age. J. Comp. Neurol. 384, 312-320.

Peters, A. (1996). Age-related changes in oligodendrocytes in monkey cerebral cortex. J. Comp. Neurol. 371, 153-163.

Peters, A., Josephson, K., and Vincent, S. L. (1991). Effects of aging on the neuroglial cells and pericytes with area 17 of the rhesus monkey cerebral cortex. J. Comp. Neurol. 229, 384-398.

Peters, A., Leahu, D., Moss, M. B., and McNally, K. J. (1994). The effects of aging on area 46 of the frontal cortex of the rhesus monkey. Cereb. Cortex 6, 621-635.

Peters, A., Morrison, J. H., Rosene, D. L. and Hyman, B. T. (1998). Are neurons lost from the primate cerebral cortex during aging? Cereb. Cortex 8 , 295-300.

Peters, A., Moss, M. B., and Sethares, C. (2000). The effects of aging on myelinated nerve fibers in monkey primary visual cortex. J. Comp. Neurol. 419 , 364-376.

Peters, A., and Rosene, D. L. (2003). In aging, is it gray or white? J. Comp. Neurol. 462, 139-143.

Peters, A., and Sethares, C. (2002). Aging and the myelinated fibers in prefrontal cortex and corpus callosum of the monkey. J. Comp. Neurol. 442, 277-291.

Peters, A., and Sethares, C. (2003). Is there remyelination during aging of the primate central nervous system? J. Comp. Neurol. 460, 238-254.

Peters, A., and Sethares, C. (2004) Oligodendrocytes, their progenitors and other neuroglial cells in the aging primate cerebral cortex. Cereb. Cortex 14, 995-1007.

Peters, A., Sethares, C., and Killiany, R. J. (2001). Effects of age on the thickness of myelin sheaths in monkey primary visual cortex. J. Comp. Neurol. 435, 241-248.

Peters, A., Verderosa, A., and Sethares, C. (2008). The neuroglial population in the primary visual cortex of the aging rhesus monkey. Glia 56, 1151-1161.

Pfefferbaum, A., Mathalon, D. H. Rawles,J. M., Zipursky, R. B., and
Lim, K. O. (1994). A quantitative magnetic resonance study of changes in brain morphology from infancy to late adulthood. Arch. Neurol. 51, 874-887.

Prineas, J. W., and McDonald, W. I. (1997). Demyelinating diseases. In Greenfield's Neuropathology, 6th Edn, D. I. Graham and P. I. Lantos, eds (London, Arnold), pp. 813-896.

Resnick, S. M., Pham, D. L., Kraut, M. A., Zonderman,A. B., and Davatzokos, C. (2003). Longitudinal magnetic resonance imaging studies of older adults: a shrinking brain. J. Neurosci. 23 , 3295-3301.

Rivers, L. E., Young, K. M., Rizzi, M., Jamen, F., Psahoulia, K., Wade, A., Kessaris, N., and Richardson, W. D. (2008). PDGFRA/NG2 glia generate myelinating oligodendrocytes and piriform projection neurons in adult mice. Nat. Neurosci. 11, 1392-1401.

Rosenbluth, J. (1966). Redundant myelin sheaths and other ultrastructural features of the toad cerebellum. J. Cell Biol. 28, 73-93.

Sandell, J.H., and Peters, A. (2001). Effects of age on the nerve fibers in the rhesus monkey optic nerve. J. Comp. Neurol. 429, 541-553.

Sandell, J.H., and Peters, A. (2002). Effects of age on the glial cells in the rhesus monkey optic nerve. J. Comp. Neurol. 445, 13-28.

Sandell, J. H., and Peters, A. (2003). Disrupted myelin and axon loss in the anterior commissure of the aged rhesus monkey. J. Comp. Neurol.466, 14-30.

Silver, M. H., Newell, K., Brady, C. Hedley-White, E. T., and Perls, T. T. (2002). Distinguishing between neurodegenerative disease and disease-free aging: correlating neuropsychological evaluations and neuropathological studies in centenarians. Psychosom. Med. 64, 493-501.

Sloane, J. S., Pietropaola, H. F. Rosene, D. L., Moss, M. B., Kemper, T., and Abraham, C. R. (1997). Lack of correlation between plaque burden and cognition in the aged monkey. Acta Neuropathol. 94, 471-478.

Smith, D. E., Rapp, P. R., McKay, H. M., Roberts, L. A., and Tuszynski, M. H. (2004). Memory impairment in aged primate is associated with focal death of cortical neurons and atrophy of subcortical neurons. J. Neurosci. 24 4373-4381.

Sobotka, S., and Ringo, J. L. (1996). Mnemonic responses of single units recorded from monkey inferotemporal cortex, accessed via transcommissural versus direct pathways: a dissociation 
between unit activity and behavior. J. Neurosci. 16, 4222-4230.

Struble, R. G., Price, D. L., Jr., Cork. L. C., and Price D. L. (1985). Senile plaques in cortex of aged normal monkeys. Brain Res. 361, 267-275.

Sturrock, R.R.(1976). Changes in neuroglia and myelination in the white matter of aging mice. J. Gerontol. 31, 513-522.

Sugiyama, I., Tanaka, K., Akita, M., Yoshida, K., Kawase, T., and Asou, H. (2002). Ultrastructural analysis of the paranodal junction of myelinated fibers in 31-month-old rats. J. Neurosci. Res. 7, 309-317.

Sullivan, E. V., Rosenbloom, M., Serventi, K. L., and Pfefferbaum, A. (2004). Effects of age and sex on volume of the thalamus, pons, and cortex. Neurobiol. Aging 25, 185-192.

Sullivan, M. V., and Hamilton, C. R. (1973a). Memory establishment via the anterior commissure of monkeys. Physiol. Behav. 11, 873-879.
Sullivan, M. V., and Hamilton, C. R. (1973b).Interocular transfer of reversed and nonreversed discrimination via the anterior commissure in monkeys. Physiol. Behav. 10, 355-359.

Tamura, E., and Parry, G. J. (1994). Severe radicular pathology in rats with longstanding diabetes. J. Neurol. Sci. 127, 29-35.

Tang, Y., Nyengaard, J. R., Pakkenberg, B., and Gundersen, H. J. G. (1997). Age-induced white matter changes in the human brain: a stereological investigation. Neurobiol. Aging 18, 609-615.

Tigges, J., Gordon, T. P., Hall. H. M., and Peters, A. (1988). Survival rate and life span of the rhesus monkey. Am. J. Primatol. 15, 263-272.

Wang, Y., Zhou, T., Ma. Y., and Leventhal, A. G. (2005). Degradation of signal timing in cortical area $\mathrm{V} 1$ and V2 of senescent monkeys. Cereb. Cortex 15, 403-408.
Waxman, S. G., Kocsis, J. D., and Black, J. A. (1995). Pathophysiology of demyelinated axons. In The Axon: Structure, Function and Pathophysiology, S. G. Waxman, J. D. Kocsis, and P. K. Stys, eds (New York, Oxford University Press), pp. 438-461.

Wilson, C. R. E., Charles, D. P., Buckley, N. J., and Gaffan, D. (2007). Fornix transection impairs learning of randomly changing object discriminations. J. Neurosci. 21, 12868-12873.

Wisco, J. J., Killiany, R. J., Guttmann, C. R. Warfield, S. K., and Rosene, D. L. (2008). An MRI study of age-related white and gray matter volume changes in the rhesus monkey. Neurobiol. Aging 29, 1563-1575.

Xi, M.-C., Liu, R.-H., Engelhardt, K. K., Morales, R. R., and Chase, (1999). Changes in the axonal conduction velocity of pyramidal tract neurons in the aged cat. Neuroscience 92 , 219-225.

Conflict of Interest Statement: The author declares that this research was conducted in the absence of any commercial or financial relationships that could be construed as a potential conflict of interest.

Received: 28 April 2009; paper pending published: 14 May 2009; accepted: 22 June 2009; published online: 06 July 2009.

Citation: Peters A (2009) The effects of normal aging on myelinated nerve fibers in monkey central nervous system. Front. Neuroanat. (2009) 3:11. doi:10.3389/neuro.05.011.2009

Copyright (C) 2009 Peters. This is an openaccess article subject to an exclusive license agreement between the authors and the Frontiers Research Foundation, which permits unrestricted use, distribution, and reproduction in any medium, provided the original authors and source are credited. 